\title{
Near Infrared Transillumination Technology as Additional Tool for Prostate Cancer Detection in vitro after Prostatectomy
}

\author{
Besarion Partsvania, ${ }^{1 *}$ Tamaz Sulaberidze, ${ }^{1}$ \\ ${ }^{I}$ Georgian Technical University, Institute of Cybernetics, Tbilisi, Georgia \\ Alexandre Khuskivadze ${ }^{2}$ \\ ${ }^{2}$ Tbilisi Medical State University, Tbilisi, Georgia \\ Levan Shoshiashvili, ${ }^{3}$ and Ketevan Chubinidze ${ }^{3}$ \\ ${ }^{3}$ Javakhishvili Tbilisi State University, Tbilisi, Georgia
}

\begin{abstract}
:
Patients with a diagnosis of prostate cancer are suggested radical prostatectomy in well known cases. After this surgical operation prostatectomy specimens usually are examined histo morphologically. The aim of this examination is detection of the cancer with high accuracy in the prostatectomy specimens and determination its aggressiveness according the Gleason score. This examination would have a significant impact on the prediction of outcomes for patients after surgical operation. The entire gland and seminal vesicles are embedded, sectioned, and examined microscopically. This often means 100-120 microscopic sections including several whole-organ sections from the central part of the gland are examined. It is evident that investigation of a histomorphological samples and detection cancerous malignancy is extremely time and labor consumptive task. In this paper we show, that near infrared radiation could be successfully used as a tool for cancerous outgrowths detection in prostatectomy specimens. Thereby, positive correlation between IR findings and macroscopic and microscopic findings could lead to higher accuracy and efficiency of histo morpoholgical investigations.
\end{abstract}

Keywords: Prostate cancer, Infrared radiation, Histo morphology

\section{INTRODUCTION}

Prostate cancer is a heterogeneous group of malignant tumors that are classified according to the World Health Organization [1].These tumors grow multifocally in the prostate and rarely produce macroscopic tumor nodules. Most adenocarcinomas are of the acinar type, which are usually referred to as prostate carcinomas $[2,3]$. When the investigation demonstrates a localized tumor and the tumor grading does not indicate aggressive carcinoma, the patient is a candidate for curative treatment such as a radical prostatectomy. Examination of prostatectomy specimens is an involved procedure for the laboratory and the pathologist [4].

The criteria used by the pathologist are architecture atypia and cellular atypia [5]. The entire gland and seminal vesicles are embedded, sectioned, and examined microscopically. This often means 100120 microscopic sections including several whole-organ sections from the central part of the gland are examined. The pathologist studies all sections in detail and describes tumor extension, grade and findings [6]. All of this information must be taken into account when planning the follow-up and deciding on additional therapy. Therefore, the ability to detect the cancer with high accuracy would have a significant impact on clinical decision making, treatment selection, and prediction of outcomes for patients [7].

Thus, it is evident that investigation of a histomorphological samples and detection cancerous malignancy is extremely time and labor consumptive task. Since human assessment is more subjective than objective and in order to reduce the error rate of diagnosis, the idea of the introduction of computer vision technique was suggested. Significant progress has been made in the use of computer vision techniques in cytology [8]. However very little work has been done in applying such techniques in histology. Consequently, seeking of efficient methods for detection of cancerous outgrowths in the prostatectomy specimens is subject of high interest. On the other hand, the use of 
Near Infrared Radiation (NIR) technology has garnered interest as a tool for intraoperative imageguided surgery in various surgical sub-disciplines for prostate [9-11].

In this paper we show, that near infrared radiation could be successfully used as a tool for cancerous outgrowths detection in prostates obtained after radical prostatectomy. Thereby, positive correlation between Infra Red (IR) findings and microscopic findings could led to higher accuracy and efficiency of hysto motpoholgical investigations.

\section{MATERIALS AND METHODS}

Experimental materials, such as prostate glands, were obtained from the radical prostatectomy. Light emitted diodes (LED)s (QT Brightek Company, USA), were utilized as the light sources, emitting an infrared light in the 850-900 nm range of the optical spectrum. The irradiated power of LEDs was low, in the range of 0,08-0,14 Watt and therefore, they did not cause any heating and damaging of the prostate tissues. To observe the prostate glands in the NIR spectrum IR-1000 Charged-Coupled Device (CCD) camera (Dage-MTI, USA) coupled with the computer was used. Prostate is placed between CCD camera and LED. Figure1.
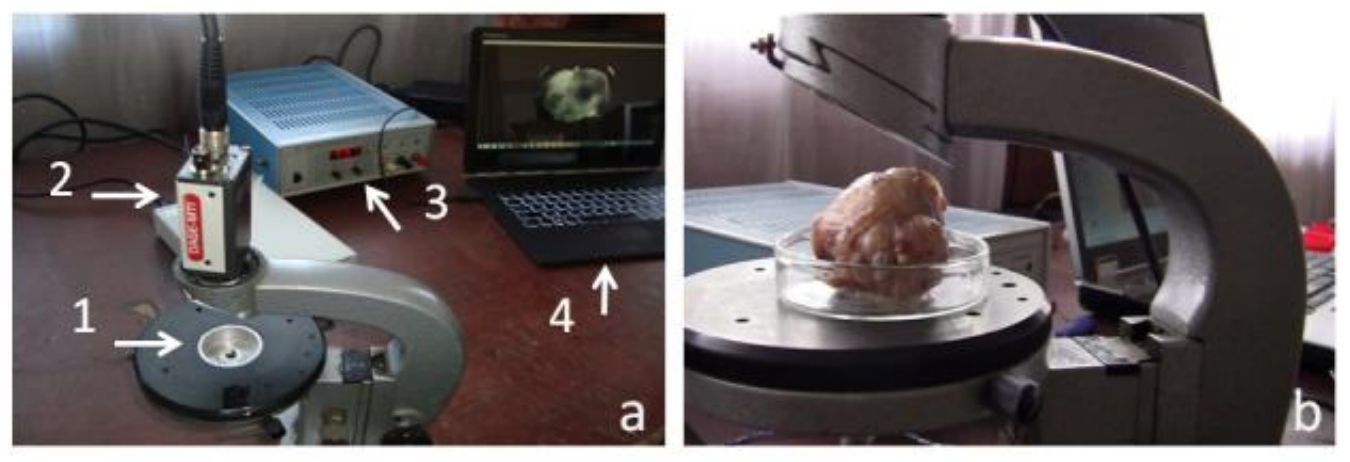

Figure1. Experimental setup. In figure $1 a$ by the arrows 1, 2, 3 and 4 are shown LED, CCD camera, power supply and computer, respectively. In the figure $1 \mathrm{~b}$ is shown prostate placed on Petri dish between CCD camera and LED. NIR light irradiation angle of LED is not less than $130^{\circ}$. CCD camera capture area covers whole surface of a prostate exposed to it, thereby no scanning is necessary.

To avoid a possible artifact caused by the visible light, experiments were carried out in the darkness. In the experiments LED was placed outside the prostate gland, from the bottom side, enabling NIR light to penetrate totally into prostate tissue.

To compare the transillumination images of non-cancerous tissues with the cancerous ones, we have elaborated software (computer program). The principle is: software divides a brightness of the NIR transillumination image into 256 degrees. Consequently, the number 255 corresponds to the absolute white image, i.e. when NIR light enters to the CCD camera through a prostate without any absorption and on the contrary, 0 corresponds to the absolute dark image, i.e. when no NIR light enters to the CCD camera, i.e. when prostate absorbs entirely NIR radiation. Then, on the NIR image potentially healthy and potentially malignant areas are marked. The software gives different codes to them. After that, software separately calculates the average values of the brightness for the potentially healthy tissue and for the malignant one, and calculates their ratio. Obtained data are stored in the memory. Such calculations are provided for all the next experiments so that, we can obtain the consequence of these ratios. When a number of experiments reach a statistically reliable value (at least 30 experiments), the software calculates a 95\% confidence interval. In our case, after the examination of 31 cancerous prostates we obtained interval of the above mentioned ratios within 0.309839-0.333541 range. Thereafter, if new prostate NIR transillumination image will be analyzed, the software will calculate above mentioned ratio of brightness. Finally, the software will compare a meaning of this calculation with that confidence interval (0.309839-0.333541). If the newly measured number occurs in this $95 \%$ confidence interval, we can declare with $95 \%$ probability that this new prostate is cancerous.

\section{RESULTS \& DISCUSSION}

Experiments revealed, that as thicker is prostate tissue as higher is an absorption of NIR light and vice versa. However brightness of one of the same thicknesses noncancerous prostate tissues NIR 
Besarion Partsvania et al. "Near Infrared Transillumination Technology as Additional Tool for Prostate

Cancer Detection in vitro after Prostatectomy"

transillumination images is nearly homogenous. Thereby different brashness in the noncancerous prostate NIR transillumination image is caused by differences in the thicknesses of the prostate tissue. In figure 2 is shown the photo and infrared NIR transillumination image of one of the noncancerous prostate.

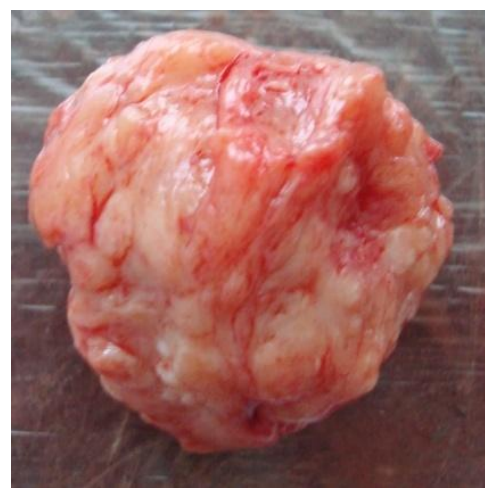

a

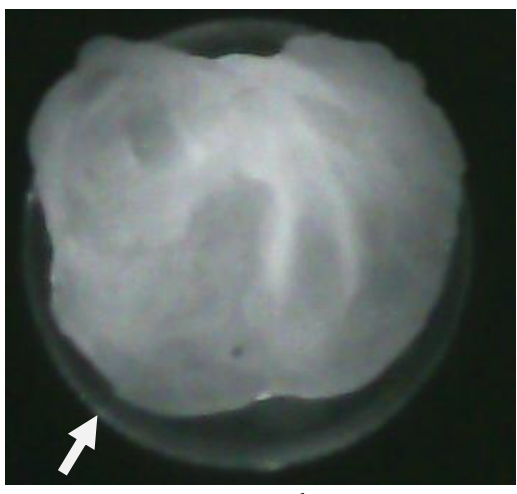

b

Figure2. Non-cancerous prostate as it seen from the rectum side. a) prostate photography. b) prostate NIR transillumination image. A transvesical (open) prostatectomy was performed after the diagnosis of benign hyperplasia (BPH). Thereby the prostate was not cancerous. A protate gland was placed on the Petri dish and an illumination was performed by using of LED with $850 \mathrm{~nm}$ vavelength. An inhomogeneity, shown by the arrow belongs to the edge of Petri dish.

After infrared examination the prostate was investigated histo- morphologically. In figure 3 is given histo-morphological picture of this prostate tissue.

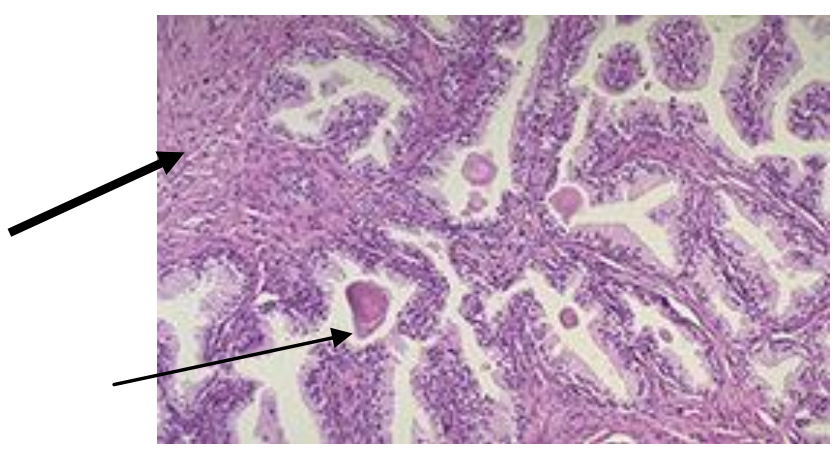

Figure3. 1) hyperplastic prostate acini, shown with thick arrow 2) fibormuscular stroma, shown with thin arrow.

Infrared image of cancerous prostate significantly differs from the noncancerous one. The brightness of the NIR transillumination image is highly nonhomogeneous. Dark areas are observed in the IR image. These areas are surrounded by relatively brighter backgrounds. In is worth mentioning that thicknesses of these different bright areas are approximately one and the same. Figure 4.

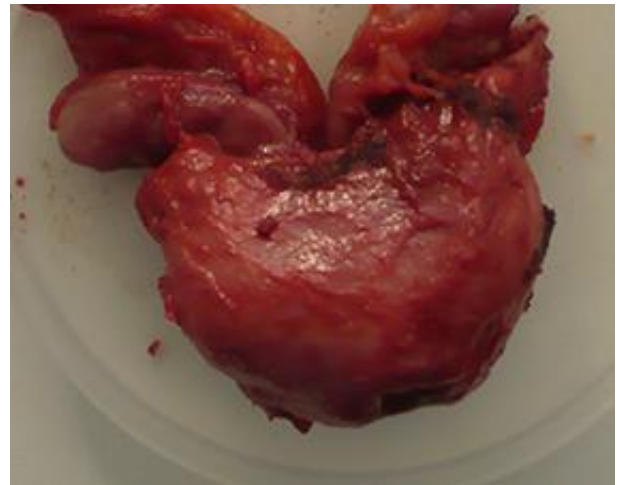

a

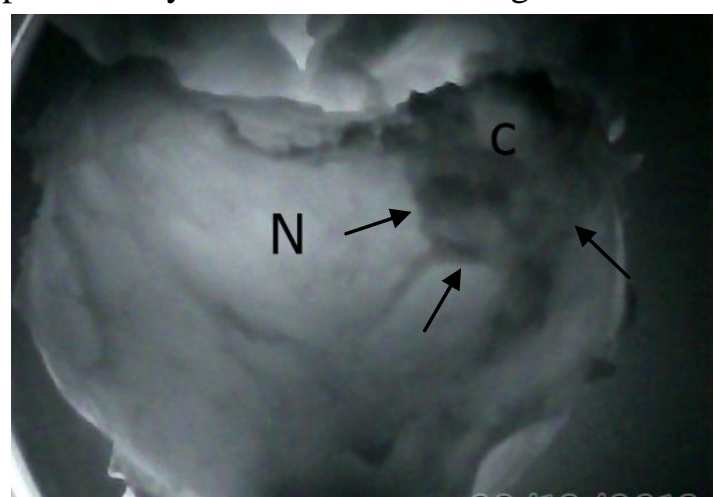

b

Figure4. Malignant prostate gland. a) prostate photography. b)- Infrared transillumination image of the same prostate. View from the rectum side. The dark area located in the right part of the NIR image (shown by arrow) corresponds to the cancerous outgrowth. This area is signed with letter "C". This area is bounded by the less darken noncancerous areas, signed with letter " $N$ ". Ratio of average brightness intensities of cancerous tissue 
to noncancerous ones was 0.322156. This occurs in 95\% confidence interval 0.309839-0.333541, thereby this prostate was cancerous with $95 \%$ probability. IR irradiation angle of used LEDs were $130^{\circ}$, thereby no scanning was necessary.

After obtaining the NIR image prostate was examined histo-morphologically. Pieces of prostate tissue were taken from both areas: cancerous, assigned with " $\mathrm{C}$ " in the figure $3 \mathrm{~b}$ and as noncancerous, assigned with "N"in the figure $3 \mathrm{~b}$ (figure 3 ). Then these pieces were examined histo-morphologically. The examination show, that in the "cancerous" piece was found adenocarcinoma with Gleason score 7 $(3+4)$, while in piece from "noncancerous" no carcinoma was found. Thereby, histo-morphological findings confirmed the results of IR examinations. In figure 5 are given, the result of the examination.

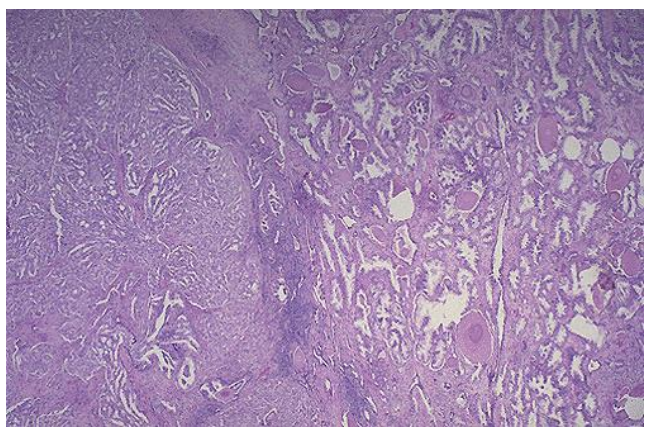

a

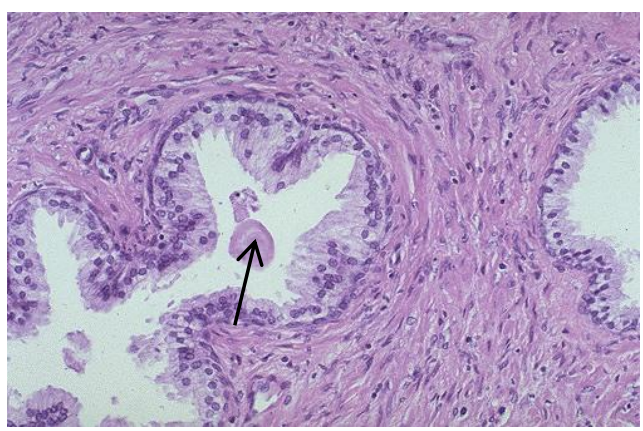

b

Figure5. a) histo-morphological view of the piece from portion tissue prostate which was determined as cancerous ("C") by IR method. In the picture clearly is observed adenocarcinoma architecture. The morphologic type is acinar type and is characterized by back-to-back proliferation of small to intermediate sized tumor acini with scant to moderate intervening stroma. b) Correspond to prostate tissue determined as not containing cancerous outgrowths (" $N$ "). The gland is well-differentiated and with intervening stroma, shown with arrow.

For discrimination of cancerous prostate infrared images with different aggressiveness is necessary to carry out statistically reliable number of experiments for each Gleason score from 6 up to 10. This conceives carrying out a significant amount of experiments to obtain statistically reliable results. In the present work we did not differentiate cancerous prostate according the aggressiveness because of a small number of experiments. We have provided 35 experiments with cancerous prostates regardless of aggressiveness. The number experiments with noncancerous prostrates was 37 . There are no infrared transillumination images of inflamed prostate tissues in present work also, because there was no prostatectomy due to this diagnosis. Experiments have shown that the brightness of malignant tissue in the transillumination image differs significantly from the brightness off healthy tissue. The Optical density of cancerous prostate tissue in the NIR range occurs much higher than the optical density of BPH tissue. Cancerous tissue absorbs more NIR energy than normal tissue. Thereby the intensity of NIR light from a prostate malignant tissue is lower in comparison with healthy one and cancerous tissue is observed as dark spots on the relatively bright background. The developed software determines intensities of brightness for both malignant and healthy tissues and gives opportunity to discriminate transillumination images of cancerous prostate tissue from noncancerous ones. Thus, NIR examination of prostatectomy specimens gives opportunity to detect the tumor location in the prostate gland in vitro. This in turn significantly reduces amount of prostate tissue slices necessary for histo morphological investigation. Thereby time necessary for histo morphological investigation will be shortened significantly and efficiency of these investigations will be significantly increased.

\section{CONCLUSION}

Infrared optical method proposed in the work can provide new insight regarding detection of tissue functional changes associated with the appearance and progression of prostate cancer.

\section{ACKNOWLEDGMENT}

This work was supported by Shota Rustaveli National Science Foundation (SRNSF), project number

AR/31/8-314/14

\section{REFERENCES}


[1] G. Sauter, J. I. Epstein, I. A. Sesterhenn, Pathology and Genetics of Tumours of the Urinary System and Male Genital Organs.. Ed. John N. Eble, Lyon, France, IARCPress, 2004. pp.159214. (Book Chapter)

[2] P. A. Humphrey, Histological variants of prostatic carcinoma and their significance, Histopathology. V 60, pp. 59-74, Jan. 2012.

[3] R. _Montironi, M. _Scarpelli, R._Mazzucchelli, A._Lopez-Beltran, M._Santoni, A. Briganti, F. Montorsi_and L. Cheng, Does prostate acinar adenocarcinoma with Gleason Score 3+3=6 have the potential to metastasize? Diagn Pathol. V 9, pp. 190-194, Oct. 2014.

[4] H. Samaratunga, R. Montironi, L. True, J. I. Epstein, D. F. Griffiths, P. A. Humphrey, T. van der Kwast, T. M. Wheeler, J. R. Srigley, B. Delahunt, L. Egevad, International Society of Urological Pathology (ISUP) Consensus Conference on Handling and Staging of Radical Prostatectomy Specimens. Working group 1: specimen handling. Modern Pathology, V 24, pp. 6-15, Jan, 2011.

[5] J. R. Srigley, Benign mimickers of prostatic adenocarcinoma, Modern Pathology, V 17, pp.328348, Mar. 2004.

[6] L. D. Thrue, Surgical pathology examination of the prostate gland. Practice survey by American society of clinical pathologists. AM J Clin Pathol, V 102, pp. 572-579, Nov, 1994.

[7] J. R. Srigley, M. B. Amin, J. I. Epstein,D. J. Grignon, P. A. Humphrey, A. A. Renshaw, T. M. Wheeler, Updated protocol for the examination of specimens from patients with carcinomas of the prostate gland. Arch Pathol Lab Med., V 130, pp. 936-946, 2006.

[8] D. Fehr, H. Veeraraghavan, A. Wibmer, T. Gondo, K. Matsumoto, H. A. Vargas,, E. Sala, H. Hricak, J. O. Deasy, Automatic classification of prostate cancer Gleason scores from multiparametric magnetic resonance images, Proc Natl Acad Sci U S A, V 112, pp.E6265-73, 2015.

[9] W. Polom, M. Markuszewski., Y. S. Rho, M. Matuszewski, Usage of invisible near infrared light (NIR) fluorescence with indocyanine green (ICG) and methylene blue (MB) in urological oncology. Part 1., Cent European J Urol, v 67, pp. 310-313, Aug. 2014.

[10] X. Wang, S.S. Huang, W.D. Heston,H. Guo, B. C. Wang, J.P. Basilion, Development of targeted near-infrared imaging agents for prostate cancer, Mol Cancer Ther., V 13, pp. 25952606, 2014.

[11] B. Zhu, G. Wu, H. Robinson, N. Wilganowski, M.A. Hall, S. C. Ghosh, K. L. Pinkston, Azhdarinia, B. R. Harvey, E. M. Sevick-Muraca, Tumor margin detection using quantitative NIRF molecular imaging targeting EpCAM validated by far red gene reporter iRFP. Mol Imaging Biol, V 15, pp. 560-568, Oct. 2013.

Citation: Partsvania B, Sulaberidze T, Khuskivadze A, Shoshiashvili L, Chubinidze K. Near Infrared Transillumination Technology as Additional Tool for Prostate Cancer Detection in vitro after Prostatectomy. International Journal of Research Studies in Medical and Health Sciences. 2016; 1(1):34-38.

Copyright: (C) 2016 Partsvania B, et al. This is an open-access article distributed under the terms of the Creative Commons Attribution License, which permits unrestricted use, distribution, and reproduction in any medium, provided the original author and source are credited. 